\title{
NET Formation in Dialysis: A Valuable, albeit Mysterious and Enticing Predictor of Mortality
}

\author{
Michael S. Goligorsky \\ New York Medical College at Touro University, Valhalla, NY, USA
}

\section{Keywords \\ Dialysis · Mortality $\cdot$ Predictors}

Compartmentalization of molecular machinery to distinct cellular organelles is an indelible strategy living organisms use for evading entropy. Here, the case in point is nuclear localization of DNA. When DNA escapes its nuclear confinement and appears extracellularly, it heralds a host of pathological conditions. In this issue of American Journal of Nephrology, Eibinder and co-workers [1] summarize their prospective studies of a representative cohort of 153 hemodialysis patients. Cell-free DNA (cfDNA) was measured at the outset and at the end of a dialysis session combined with the 46-month follow-up. The investigators document that the post-dialysis cfDNA levels constitute a powerful predictor of all-cause mortality at 1-3 years with odd ratios of $4.6,4.3$, and 6.2 , respectively. The predictive value of this parameter was superior to several other well-characterized predictors, thus making the post-dialysis cfDNA the most sensitive marker of lethal outcomes. The clinical significance of this study is hard to overestimate, as it alerts physicians to concealed intrinsic problems in need to be addressed. This study buttresses the previous pilot investigation by the same group and furthers 2 other investigations re-

karger@karger.com

(c) 2020 S. Karger AG, Basel

www.karger.com/ajn

Karger! porting similar results [2-4]. Collectively, these innovative investigations not only advance the field of biomarkers for long-term morbidity and mortality but also importantly beacon further exploration of causes behind elevated post-dialysis cfDNA.

In general, the sources of cfDNA are threefold: apoptosis, necrosis, and NETosis (the latter stands for neutrophil extracellular traps formation). Assuming uremic toxins increase cellular apoptosis and necrosis, it goes against the reason to ascribe post-dialysis, ergo after clearance of some uremic toxins, elevation of cfDNA to these causes. Yet, a well-known destruction of some nucleated formed elements during hemodialysis could contribute to elevation of cfDNA. However, the observation that cfDNA is elevated in patients on peritoneal dialysis as well (reviewed in [5]) casts doubt on the hypothesis that destruction or NETosis of activated neutrophils on dialysis membranes is a major contributor to elevated cfDNA in hemodialysis. For the sake of argument, even with the destruction of all neutrophils, DNA release will be orders of magnitude lower that the observed concentrations of cfDNA in dialysis patients (see below).

NET formation, an evolutionarily conserved process of entrapment of circulating bacterial or viral invaders, most recently reviewed by Burgener and Schroder [6], 
Fig. 1. Pictorial summary of the triggers of NET formation. Neutrophil activation by infectious and noninfectious mechanisms leads to NET formation and elevated levels of cfDNA. cfDNA is degraded by DNases. Maneuvers leading to the activation of adenosine A2A and elevation of cAMP have been shown to inhibit NET formation [7]. The list of noninfectious triggers of NET formation is now expanded to include hemodialysis. In addition, I would speculate that hemodialysis may also affect the process of degradation of cfDNA. Neutrophils activation by infectious and noninfectious mechanisms leads to NET formation and elevated levels of cfDNA. cfDNA is degraded by DNases. Maneuvers leading to the activation of adenosine A2A and elevation of cAMP have been shown to inhibit NET formation [7]. cAMP, cyclic AMP; cfDNA, cell-free DNA.

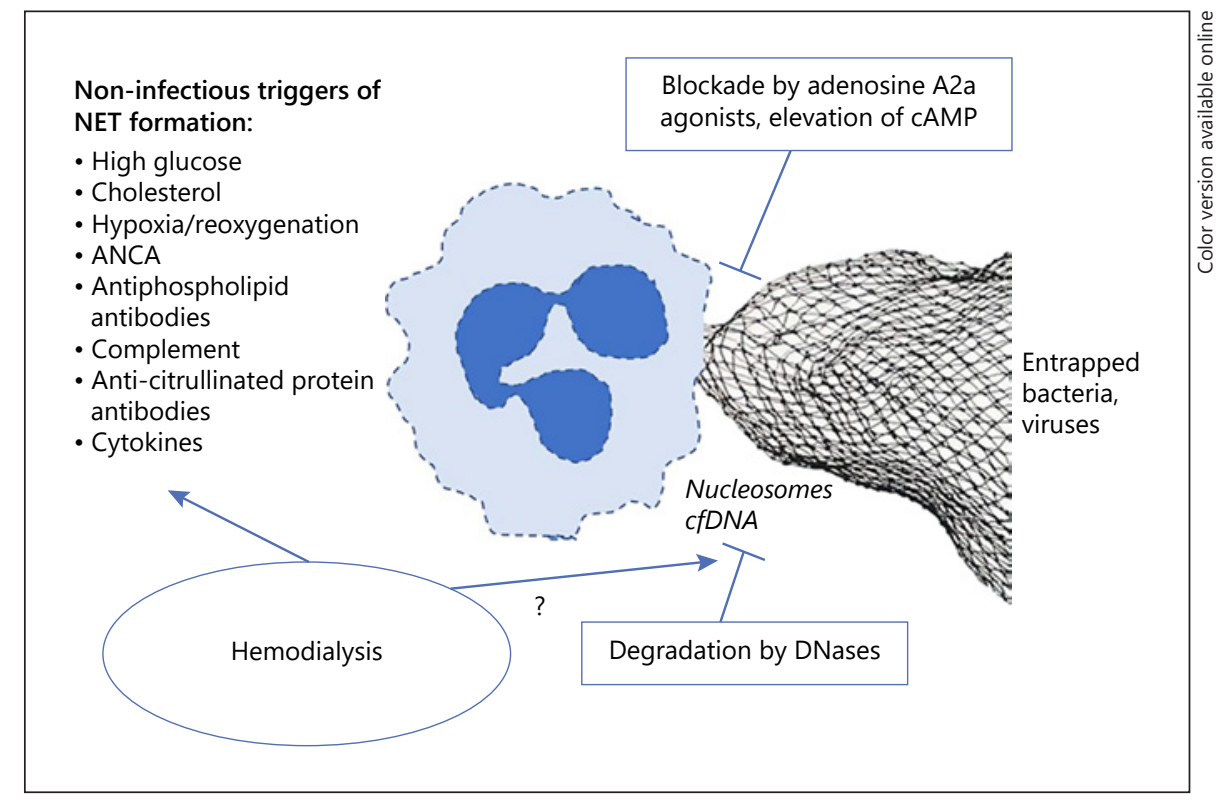

can be triggered by NADPH oxidases and reactive oxygen species (suicidal NETosis), by activation of caspase-4/11, gasdermin $\mathrm{D}$ in the process resembling pyroptosis (noncanonical NETosis) or activation of Toll-like receptors-2 and 4 (vital NETosis). Yet, non-bacterial triggers have been identified, such as high glucose, cholesterol, complement C5a, hypoxia, cytokines, activated platelets, ANCA, anti-citrullinated protein antibodies, antiphospholipid antibodies, among others (Fig. 1), thus expanding NET formation to autoimmune disorders, atherosclerosis, diabetes, ischemia-reperfusion injury, and cancer pathophysiology [7]. The list of non-infectious triggers of NET formation is now expanded to include hemodialysis. In addition, I would speculate that hemodialysis may also affect the process of degradation of cfDNA.

Indeed, NET components (decondensed chromatin backbone consisting of nucleosomes - DNA coiled around histones) are present in hemodialysis patients [3], thus strongly implicating this process in ESRD-associated atherogenesis, endothelial dysfunction, and thrombosis in the absence of bacterial invaders. It remains puzzling; however, that post-dialysis elevation of cfDNA has superior predictive value over its pre-dialysis value and other markers of morbidity and mortality.

Degradation of NETs is accomplished by DNases present in plasma and in macrophages phagocytosing these structures. It has been detected that SLE or cystic fibrosis patients exhibit elevated levels of cfDNA (the levels in the sputum of cystic fibrosis patients were reported to reach an astounding concentration of 3-14 mg/mL) accompa- nied by the reduced abundance and/or activity of DNase [8]. Based on these findings, it is not surprising that recombinant human DNase I used as a nebulizer brings symptomatic relief to some patients with cystic fibrosis. Similarly, many cancer patients exhibit increased plasma cfDNA and reduced DNase level and/or activity, leading to clinical trials of recombinant human DNase I in various malignancies.

In my opinion, however, it would be difficult to attribute elevated cfDNA in post-dialysis blood exclusively to either of the above causes - apoptosis, necrosis, or NETosis. A simple balance sheet of total genomic DNA per cell being in the order of 6-7 pg, the number of circulating neutrophils, against the half-life of cfDNA being in the range of 10-15 $\mathrm{min}$, and DNase constitutively present in the circulation, it becomes obvious that elevated levels of cfDNA are not exclusively a function of increased release of cellular DNA rather are significantly impacted by its impaired degradation by the DNases. Intriguingly, Kim et al. [4] observed, to the contrary, that patients with highest DNase levels show increased risk of cardiovascular events, both nonfatal and fatal. These investigators, however, emphasized that the abundance of this enzyme is not necessarily proportional to its activity, implying that reduced DNase activity could potentially contribute to elevated levels of cfDNA. Examples of suppressed DNase activity accompanied by elevated cfDNA are many - SLE, cystic fibrosis, and cancers. One wonders whether it could happen that dialysis is associated with an inhibition of DNase activity or makes cfDNA more resistant to its en- 
zymatic degradation. If that is the case, the mystery of present findings on a valuable prognostic marker could gain pathogenic understanding. Notwithstanding the persistent mechanistic puzzlement, the entire field of NET formation in renal disease, uremia, and dialysis deserves further scrutiny, as it has a potential to disclose novel biomarkers, as well as deepen our understanding of diverse comorbidities in kidney injury.

\section{Acknowledgement}

Studies in the author's laboratory are supported in part by NIH grant NIH HL144528 and New York Community Trust grant for Research and Education.

\section{Conflict of Interest Statement}

There are no conflicts of interest.

\section{References}

1 Eibinder Y, Shnaider A, Ghanayem K, Basok A, Rogachev B, Lior Y, et al. Elevated circulating cell-free DNA in hemodialysis-treated patients is associated to increased mortality. Am J Nephrol. 2020. DOI: 10.1159/000510771.

2 Tovbin D, Novack V, Wiessman MP, Abd Elkadir A, Zlotnik M, Douvdevani A. Circulating cell-free DNA in hemodialysis patients predicts mortality. Nephrol Dial Transplant. 2012;27(10):3929-35.

3 Kim JK, Park MJ, Lee HW, Lee HS, Choi SR, Song YR, et al. The relationship between au- tophagy, increased neutrophil extracellular traps formation and endothelial dysfunction in chronic kidney disease. Clin Immunol. 2018;197:189-97.

4 Kim JK, Lee HW, Joo N, Lee HS, Song YR, Kim HJ, et al. Prognostic role of circulating neutrophil extracellular traps levels for longterm mortality in new end-stage renal disease patients. Clin Immunol. 2020;210:108263.

5 Korabecna M, Tesar V. NETosis provides the link between activation of neutrophils on hemodialysis membrane and comorbidities in dialyzed patients. Inflamm Res. 2017;66(5): 369-78.

6 Burgener SS, Schroder K. Neutrophil extracellular traps in host defense. Cold Spring Harb Perspect Biol. 2020;12(7):a037028.

7 Sorvillo N, Cherpokova D, Martonod K, Wagner D. Extracellular DNA NET-works with dire consequences for health. Circ Res. 2019;125:47-488.

8 Hawes MC, Wen F, Elquza E. Extracellular DNA: a bridge to cancer. Cancer Res. 2015; 75(20):4260-4. 